\title{
Reply to Comment by J. Zhang and N. Makris on "Estimates of the Ground Accelerations at Point Reyes Station during the 1906 San Francisco
}

\author{
Earthquake" by A. Anooshehpoor, T. H. Heaton, B. Shi, and J. N. Brune
}

\author{
by A. Anooshehpoor, T. H. Heaton, B. Shi, and J. N. Brune
}

Contrary to the comments by Zhang and Makris (hereafter, ZM), our equations of motion governing the rocking response of a rectangular block subjected to a full-sine acceleration pulse are correct. Therefore, the first part of ZM's discussion, which is based primarily upon the assumption that the equations of motion in our article were incorrect, is inappropriate. In the second part of the discussion, ZM present new results for mode 2, toppling without impact. We did not consider this mode because it was not relevant to the Point Reyes train, which by eyewitness accounts, had overturned after experiencing one impact. However, as explained in this reply, toppling with no impact is never the minimum condition for overturning, and would in general involve very large horizontal accelerations, especially at frequencies where mode 2 is the only overturning mode.

As ZM correctly point out, the sign of the second term on the right-hand side of equation (3) in our article must be negative. However, this error (not present in our first paper on rocking motion, Shi et al., 1996) was not reflected in the linearized equations (5) and (7). In Figures 4 and 5, we have plotted the "inertial acceleration" of the center of mass whereas the term "ground acceleration" is used in both the legends and the figure captions. ZM are also correct that the value of $\eta$ in the captions of Figures 6 and 8 should have been 0.9 instead of 0.95 .

In describing rocking motion of a two-dimensional rigid block one in general needs to use two separate equations of motion, one about each of the two rocking points. Equations (5) and (7) in our article describe the rocking motion about $O_{1}$ and $O_{2}$, subject to the input motion specified in equation (1). These two equations are correct, and are identical to equations (4) and (5) in ZM's comment. These simple equations were obtained from the Newton's second law of motion. They relate the rate of change of the angular momentum, $\vec{L}$, to the total torque:

$$
\begin{aligned}
\frac{d \vec{L}}{d t} & =\sum_{i} \vec{\tau}_{\mathrm{i}} \\
& =\vec{R} \times m \vec{g}+\vec{R} \times m \vec{a}
\end{aligned}
$$

The first term on the right-hand side is the restoring torque due to the gravitational force; and the second term is the inertial torque in a noninertial reference frame.
Equation (5) in our article is the linearized equation of motion about $O_{1}$ for the time interval prior to the impact $(0$ $\left.\leqq t \leqq t_{\mathrm{i}}\right)$. After the impact $\left(t>t_{\mathrm{i}}\right)$, the motion is governed by equation (7). Equation (7) was written by assuming that the motion about $\mathrm{O}_{2}$ is the mirror image of the motion about $O_{1}$. Therefore, one can use equation (5) along with the proper initial conditions (i.e., at $t=t_{\mathrm{i}}, \theta_{2}=0$, and $\dot{\theta}_{2}=$ $\left.-\eta \dot{\theta}_{1}\right)$ to describe the motion after the impact with the exception that the inertial term will have an opposite sign $(\vec{a} \rightarrow-\vec{a})$. In constructing equation (7) we did not make any assumption about the sign of $\theta_{2}$. However, if we had assumed a negative $\theta_{2}$, this equation would have become identical to equation (5) of ZM's discussion through the substitution $\theta_{2}=-\theta_{2}$.

Figures 4 and 5 in our article display the solutions of separate equations of motion on the same plot. Thus, it is a matter of convention how one, at $t=t_{\mathrm{i}}$, ties these solutions (before and after the impact) together. According to the convention used in our article, angular displacement is positive about both rocking axes. However, in preparing Figures 4 and 5 of our article, we reversed the polarity of the angular displacement after the impact with the intention to make them easier to read. We did not do the same with the angular velocity because we wished to emphasize the discontinuity of motion during impact. We now realize that this has caused some confusion because, after the impact, the angular velocity curve does not appear to represent the time derivative of the angular displacement. In retrospect, we should have either left the polarity of $\theta\left(t>t_{\mathrm{i}}\right)$ unchanged (e.g., Figure 1 in this reply), or reversed the polarity of $\dot{\theta}\left(t>t_{\mathrm{i}}\right)$, as well. The latter choice would have made Figures 4 and 5 in our article identical to Figures 2 and 3 in ZM's discussion, respectively.

As a result of ZM's discussion we carefully checked our algorithm. We found three additional typographical errors. They are (1) in equation (21), on the last line, the factor $-\eta$ is missing; (2) in equation (24) and the following line $\tau$ should be replaced by $t_{\mathrm{i}}$; and (3) the first line below equation (25) should say "with $t_{\mathrm{i}}$ defined by $\theta_{3}\left(t_{\mathrm{i}}\right)=0$."

By comparing our results with the numerical results presented in ZM's comment, we also discovered a graphical error in plotting the minimum condition described by equation (25). Although equation (25) is correct we found that in the plotting program $\tau$ was erroneously substituted for $t_{\mathrm{i}}$. 
$\omega / p=8, \quad A=8.64 \alpha g, \quad \eta=0.95, t_{i} / \tau=2.06$

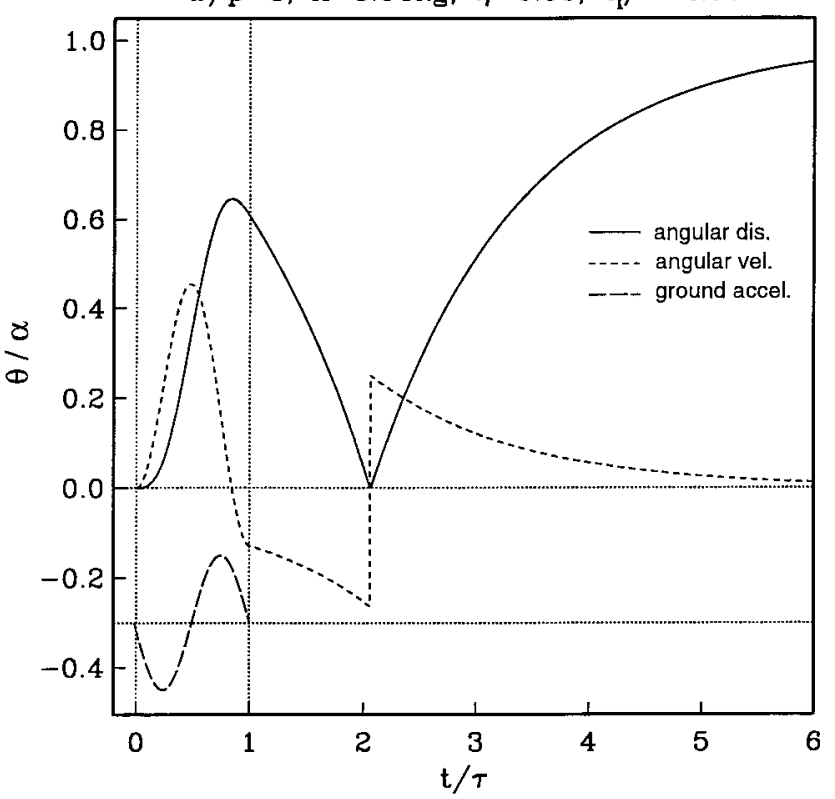

Figure 1. A plot of the normalized angular displacement $\theta / \alpha$ (Equation (22) in the original article) versus dimensionless time $t / \tau$ for $w / p=8$ and $\eta=$ 0.95 . The the angular velocity of the block and the ground acceleration are also given on the same plot. This is a replot of Figure 5 in the original article where the polarity of the angular displacement for $t>t_{\mathrm{i}}$ was reversed for plotting purposes.

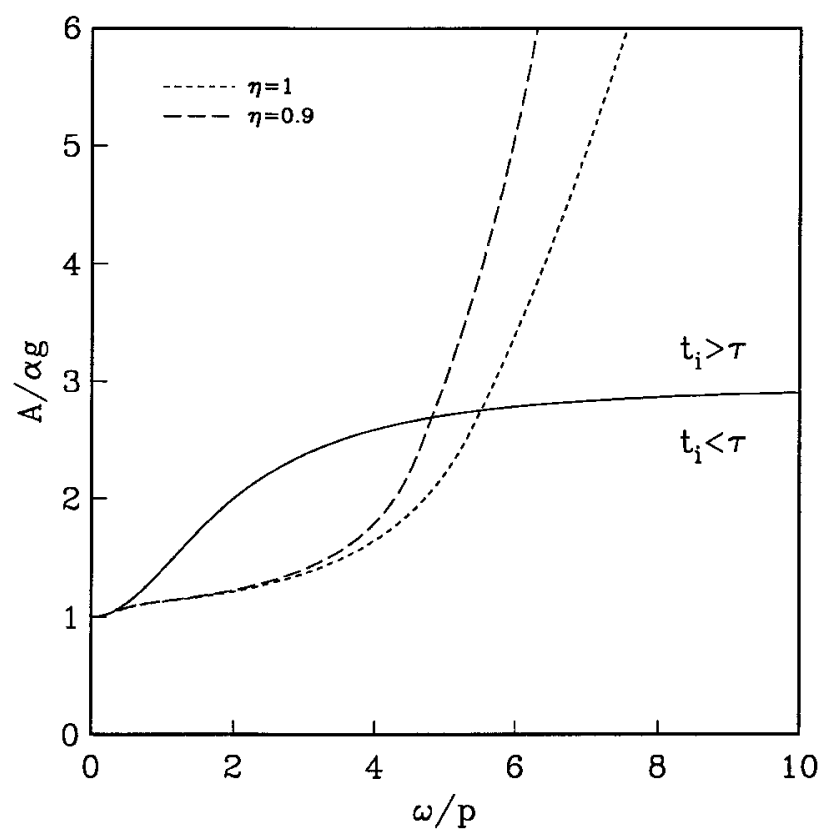

Figure 2. This is a re-plot of Figure 6 in the original article with the graphical error for $\eta=0.9$ case corrected. This graphical error was carried over in plotting Figures 7 and 8 in the original article.
This plotting error did not affect the curve for $\eta=1$ because the term containing $t_{\mathrm{i}}$ vanishes. However, it did slightly affect the curve for $\eta=0.9$ for $t_{\mathrm{i}}>\tau$ in Figures 6-8 in our original article. The corrected results are plotted in Figures 2 and 3 of this reply. This correction increases the estimated toppling acceleration at $2 \mathrm{~Hz}$ from $1.05 \mathrm{~g}$ to $1.20 \mathrm{~g}$.

The main objective of our article was to provide lowerbound estimates for ground accelerations that had toppled the train. We did not consider ZM's mode 2 because according to the conductor, the train was toppled after experiencing one impact. At the beginning of our original article we explicitly described the problem that we were to study. In studying this problem, the main purpose in seeking an analytical solution was to gain a better understanding of the physics involved. We were aware that ground motions during the earthquake were much more complex than a simple sine-wave pulse, and thus we used a numerical technique to calculate the response of the train to realistic accelerograms.

Figures 6 and 7 in ZM's comment present complete solutions for the rocking response subject to a full sine-wave pulse of acceleration. They show results that we did not consider in our study where we limited the problem to obtaining the minimum condition for overturning with one impact. Although we believe ZM's results for mode 2 are important from the theoretical point of view, they are not important for

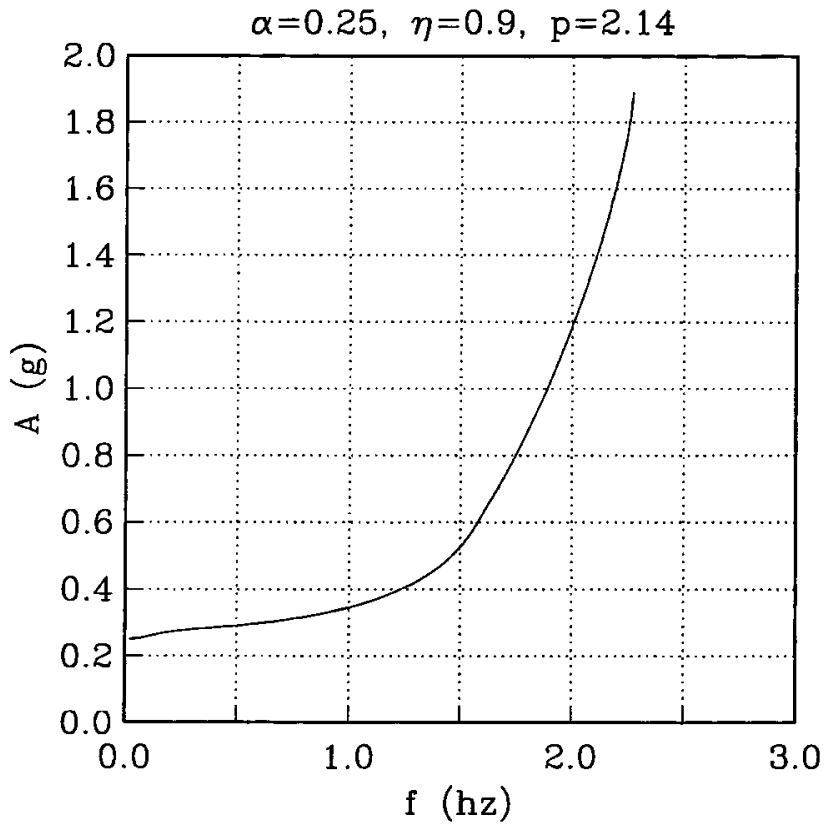

Figure 3. This is the corrected version of Figure 8 in the original article, which shows the minimum toppling amplitude of a full sine wave of ground acceleration that could topple the narrow-gauge locomotive at Point Reyes Station with one impact. 
the response of precarious rocks studied in our earlier paper (Shi et al., 1996), nor in our study of the Point Reyes train. For instance, according to Figure 7 in ZM's discussion, at about $2.7 \mathrm{~Hz}$ the peak ground acceleration necessary to topple the train with mode 2 is about $4 g$, higher than any acceleration in the Frankel et al. (1996) $2 \%$ in 50 years hazard map.

As pointed out in our earlier paper (Shi et al., 1996), because of the strong nonlinearity of the problem, there could be in general a zone of accelerations above the minimum toppling acceleration for any $w / p$, where toppling does not occur for particular accelerations. This zone was also pointed out by Yim et al. (1980). In general, for any state of $\theta$ and $\dot{\theta}$, we can always find a special subsequent ground motion which will prevent the object from toppling, but the implied accelerations might be totally unrealistic.

In summary, we would like to thank ZM for their comments. We are pleased that their numerical calculation has confirmed our analytical solutions stated in equations (13) and (22). We regret the errors in our article. However, none of these errors affect any of the conclusions in either the current article (Anooshehpoor et al., 1999) or Shi et al. (1996).

\section{References}

Frankel, A., C. Mueller, T. Barnhard, D. Perkins, E. V. Leyendecker, N. Dickman, S. Hanson, and M. Hopper (1996). National Seismic Hazard Maps, June 1996, U. S. Department of Interior, U. S. Geological Survey.

Shi, B., A. Anooshehpoor, Y. Zeng, and J. N. Brune (1996). Rocking and overturning of precariously balanced rocks by earthquakes, Bull. Seism. Soc. Am. 86, 1364-1371.

Yim, C. S., A. K. Chopra, and J. Penzien (1980). Rocking response of rigid blocks to earthquakes, Earthquake Engineering and Structural Dynamics 8, 565-587.

Seismological Laboratory 174

Mackay School of Mines

University of Reno

Reno, Nevada 89557-0141

(A. A., B. S., J. N. B.)

California Institute of Technology

Dept. of Civil Engineering, MS 104-44

Pasadena, California 91125

(T. H. H)

Manuscript received 13 December 1999. 